\title{
Oststudien besser als ihr Ruf
}

\author{
Qualitativ hochwertige klinische Studien kommen aus den \\ USA oder zumindest aus Westeuropa. Wurden die Studien \\ dagegen in den ehemaligen Ostblockländern durchgeführt, ist \\ das Ergebnis mit Vorsicht zu genießen - ein Vorurteil?
}

\begin{abstract}
- in Kardiologe mit Anstellungen in - Russland und Schweden hat sich die Mühe gemacht, die Verlässlichkeit von Studien, die in Russland und anderen ehemaligen Ostblockländern durchgeführt wurden, unter die Lupe zu nehmen. Er kommt zu dem Schluss, dass die erzielte Datenqualität westlichen Studien durchaus vergleichbar ist. Bei der Einhaltung ethischer Standards sowie der Beachtung der Good Clinical Practice müssten die Oststudien den Vergleich ebenfalls nicht scheuen. Auch zahlreiche Inspektionen der US-amerikanischen Food and Drug Administration bescheinigen osteuropäischen Prüfzentren eine überdurchschnittliche Qualität. Ein klarer Vorteil ist schließlich, dass es in diesen Ländern häufig einfacher ist, rasch ausreichend große
\end{abstract}

Patientenzahlen zu rekrutieren und Patienten zu finden, die bislang unbehandelt waren, falls das Studienprotokoll dies erfordert.

Fazit: Vielleicht rümpfen wir zu Unrecht die Nase, wenn wir eine Oststudie in die Hände bekommen. Unsere Kollegen im Osten standen bisher in dem Ruf, klinische Studien zu Dumpingpreisen anzubieten und "auf die Schnelle“ durchzuführen. Dass unter solchen Voraussetzungen die Qualität zu leiden hatte, war eine fast automatische Schlussfolgerung. Die hier vorgelegte und recht überzeugende Analyse legt dagegen nahe, dass sogar eher das Gegenteil der Fall ist.

Prof. Dr. Edzard Ernst, Exeter

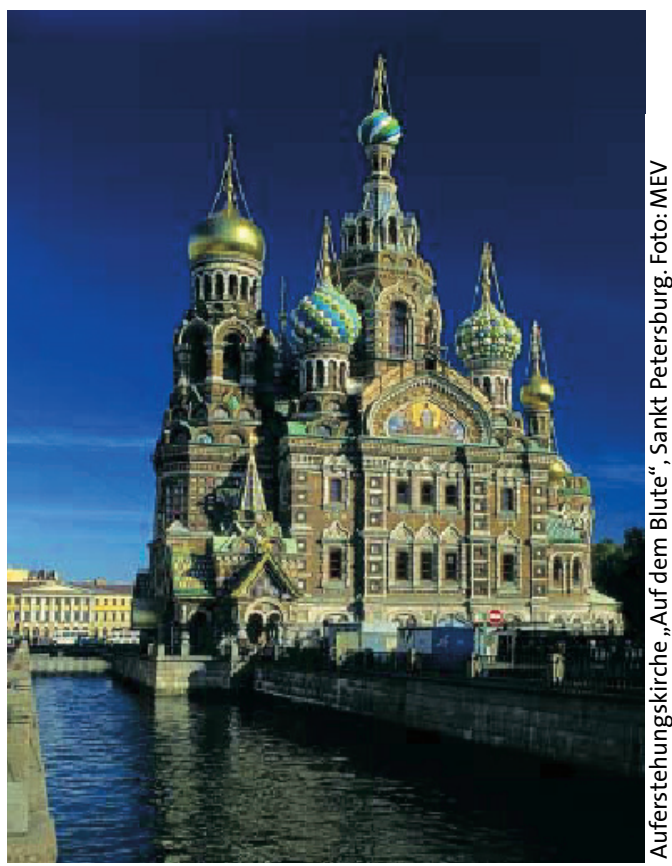

In Osteuropa wird billig gearbeitet, aber auch gut?

Platonov P. Clinical trials in Russia and Eastern Europe: recruitment and quality. Int J Clin Pharmacol Ther 2003; 41: $277-80$

\section{Das Problem mit dem zu kleinen "n“}

\begin{abstract}
Wenn Ergebnisse von klinischen Studien „nicht signifikant“ sind, muss man sich offensichtlich fragen, ob wirklich das getestete Therapieverfahren nichts bringt oder ob schlicht das Studiendesign insuffizient ist.
\end{abstract}

E in Statistiker vom Memorial Sloan Kettering Cancer Center in New York hat einen einfachen, aber vielsagenden Vergleich angestellt. Er identifizierte klinischen Studien aus den vier Fachzeitschriften Lancet, New England Journal of Medicine, Journal of the American Medical Association und American Journal of Medicine, die Berechnungen der notwendigen Fallzahl enthielten. Er verglich sodann die Standardabweichung der Zielvariablen, die in diese Berechnung eingegeben worden war, mit der tatsächlichen Standardabweichung, die sich aus den Studienergebnissen ergab. Er stellte eine deutli- che Unterschätzung dieser Variable bei 80\% der Studien fest. Bei einem Viertel der Studien wäre sogar eine mehr als viermal so große Probandenzahl notwendig gewesen, um das Ergebnis mit der ursprünglich geplanten Sicherheit bestimmen zu können. Insgesamt zieht der Autor den Schluss, dass klinische Studien - selbst solche aus den renommierten Journalen - häufig zu kleine Fallzahlen aufweisen, um einen Theraren.

Fazit: Wenn klinische Studien mit zu wenig Patienten durchgeführt werden, pieeffekt mit Sicherheit zu identifizie- laufen sie Gefahr, einen therapeutischen Effekt zu übersehen, obwohl ein solcher vorhanden ist. Dies nennt sich im Fachjargon der Statistiker „Fehler zweiter Art“. Formale Fallzahlsberechnungen haben die Aufgabe, Derartiges $\mathrm{zu}$ vermeiden. Für die Berechnung muss die oben genannte Standardabweichung geschätzt werden. Die hier vorgelegte elegante Analyse zeigt, dass diese Schätzung meist falsch und zugunsten kleiner Fallzahlen ausfällt. Dies ist zunächst nur allzu verständlich: Hohe Fallzahlen verteuern die ohnehin schon kostspieligen Studien. Andererseits bedeuten zu kleine Fallzahlen, dass viele Studien aus wirksamen Therapien fälschlicherweise unwirksame Behandlungsformen machen.

Prof. Dr. Edzard Ernst, Exeter

Vickers AJ. Underpowering in randomized trials reporting a sample size calculation. J Clin Epidemiol 2003; 56: 717-20 